\title{
Covid-19: retos y oportunidades en el proceso de acreditación de las universidades
}

\section{Covid-19: challenges and opportunities in the university accreditation process}

DOI: $10.46932 / \mathrm{sfjdv2n2-045}$

Received in: january 1st, 2020

Accepted in: March 30th, 2020

\author{
Pedro Julián Ormeño Carmona \\ Doctor \\ Institución actual: Universidad Nacional De Ucayali \\ Dirección completa: Car. Federico Basadre Km. Ref: Ucayali Coronel Portillo Calleria \\ Correo electrónico: pedro_ormeno@unu.edu.pe \\ Manuel Rocha Gonzales \\ Doctor \\ Institución actual: Universidad Nacional De Ucayali \\ Dirección completa: Car. Federico Basadre Km. Ref: Ucayali Coronel Portillo Calleria \\ Correo electrónico: manuel_rocha@unu.edu.pe

\section{José Ángel Meneses Jiménez \\ Doctor} \\ Institución actual: Universidad Nacional De Ucayali \\ Dirección completa: Car. Federico Basadre Km. Ref: Ucayali Coronel Portillo Calleria \\ Correo electrónico: jose_meneses@unu.edu.pe
}

\section{RESUMEN}

Estando próximos al cumplimiento del bicentenario del país, es necesario reflexionar sobre esta frase: "La educación que queremos para el Perú" que subtitula el Proyecto Educativo Nacional al 2021, y que se relaciona con el título de la presente investigación (Consejo Nacional de Educación-Perú (2007)

El objetivo de la investigación fue establecer los retos y oportunidades que se están presentando durante el proceso de acreditación en el estado de emergencia.

La metodología de estudio es de tipo investigación-acción, pues conto con una intervención en la práctica profesional con la intención de ocasionar una mejora. Sunivel es aplicativa, pues se centró en encontrar mecanismos que permitan lograr un objetivo.

Los resultados y conclusiones, fueron que los retos que se tienen frente a este proceso giran en torno al proceso de transición de lo presencial sincrónico, a lo virtual mixto sincrónico y asincrónico.

Las oportunidades, que nos ha traído esta pandemia son la mayor de inversión, y que eran necesarias para el cumplimiento del proceso de acreditación, las cuales se describen en la Dimensión 3: Soporte Institucional; Factor 10: Infraestructura y soporte, Estándar 30 Sistema de información y comunicación y el estándar 31 Centros de información.

La conclusión final es que la actual situación que vive la academia a consecuencia del COVID 19, va permitir implementar una reingeniería del proceso de enseñanza aprendizaje.

Palabras clave: COVID 19, Calidad Académica, Acreditación 


\section{ABSTRACT}

Being close to the country's bicentennial, it is necessary to reflect on this phrase: "The education we want for Peru" that subtitles the National Education Project to 2021, and which is related to the title of this research (Consejo Nacional de Educación-Perú (2007).

The objective of the research was to establish the challenges and opportunities that are being presented during the accreditation process in the state of emergency.

The study methodology is of the action-research type, since it involved an intervention in professional practice with the intention of bringing about an improvement. Sunivel is applicative, since it focused on finding mechanisms to achieve an objective.

The results and conclusions were that the challenges facing this process revolve around the process of transition from the face-to-face synchronous to the virtual, mixed synchronous and asynchronous.

The opportunities that this pandemic has brought us are the greatest investment, and that were necessary for the fulfillment of the accreditation process, which are described in Dimension 3: Institutional Support; Factor 10: Infrastructure and Support, Standard 30 Information and Communication System and Standard 31 Information Centers.

The final conclusion is that the current situation of the academy as a result of COVID 19 will allow the implementation of a reengineering of the teaching and learning process.

Key words: COVID 19, Academic Quality, Accreditation.

\section{INTRODUCCIÓN}

Uno de los objetivos fundamentales de las instituciones académicas, es la formación del recurso humano en las áreas que le son establecidas, ahora bien; las Universidades en su quehacer diario, como es el impartir conocimiento busca el reconocimiento público y esto solo es logrado a través de la acreditación, que no es más que el examen a la alta calidad educativa, al respecto; las Universidades para lograr este cometido deben ser sometidas y pasar por una rigurosa y profunda evaluación, todo ello; con el fin de comprobar si cumple con los estándares, las normas y reglas exigidas para la formación de los estudiantes y los futuros profesionales.

En tal sentido; la acreditación Universitaria permite cotejar la educación impartida, en dónde; se pretende examinar las dinámicas presentes en dichos estudios, poder mejorar la calidad y plantearse las metas establecidas, la acreditación por lo general son decisiones voluntarias. Lo más importante de esto, es lograr certificar la calidad de instrucción de la institución o del programa educativo establecido, ésta autoevaluación son validados por expertos externos, que luego de que es validada, tiene un lapso de tiempo específico, al respecto; las acreditaciones que son emitidas a una institución de educación superior, lo son por un tiempo limitado, puesto que; estas deber ser revisadas y evaluadas cada cierto tiempo.

Sin duda alguna; la acreditación en las instituciones universitarias significan retos que deben afrontar para lograr la excelencia académica, sin embargo; ha sucedido un hecho que ha trastocado la vida institucional y académica de los entes encargados de la educación en la mayoría de los países del mundo, la crisis generada por el Covid-19, la cual; ha cambiado la forma de gestión educativa, produciendo un 
sismo al cual no se estaba acostumbrado, "el paso de una educación tradicional o educación presencial a una educación virtual o a distancia”, en donde el contacto entre los discentes y los docentes se redujo al mínimo necesario, estos cambios están obligando a los encargados de impartir conocimiento, a repensar la forma en que se venía aplicando la educación, con nuevas modalidades, ya no, de forma parcial sino de manera total, la pregunta es ¿ qué parámetros se deben establecer para medir la excelencia académica, en tiempos de educación virtual producto de la crisis generada por el Covid-19? ¿Cuál será el método utilizado para la acreditación institucional Universitaria, en una educación a distancia?

$\mathrm{Al}$ respecto; la educación a distancia es de vieja data y tiene varios precedentes, el ejemplo clásico lo representan los primeros cursos por correspondencia que se impartieron en los EEUU a comienzo del siglo XVIII. Luego; a mitad del siglo IX es fundado e introducido el primer curso de idiomas por correspondencia en ese mismo país, lo que fue aprovechado por Europa para impartir educación por correo postal, igualmente; en el siglo XX, con la invención de la radio se empieza a dar educación por radio a los niños, así como; para la educación Universitaria.

La revolución de las TIC en los años 80 del presente siglo, significó un verdadero hito, que cambiaría la percepción de la educación, tal como se conoce hoy en día y es que, la llegada del internet ha estimulado la educación virtual o educación en línea.Sin duda alguna; el desarrollo de las TIC, está cimentando las bases para un verdadero cambio de paradigma, como es la educación a distancia, en dónde; las estructuras físicas quedan relegadas a un segundo plano, ya no se necesita de grandes inversiones en obras de infraestructura para albergar a los miles de estudiantes que cada día, necesitan trasladarse e ir a esos centros de estudios. La crisis generada por el Covid-19, ha dejado al descubierto una nueva realidad: las estructuras físicas son obsoletas, son los nuevos retos a los que se enfrentan los centros e instituciones dedicadas a la noble tarea de educar de manera tradicional, la pandemia está causando una verdadera revolución educativa, los estudiantes ya no tienen la necesidad de trasladarse a las instituciones de educación superior a recibir clases, solo basta conectarse a la red, desde su casa, su lugar de trabajo o desde cualquier lugar del mundo y hacer un clic.

Evidentemente; el uso de las redes informáticas significó un “Boom”para la educación en general, puesto que; esta ha sido aprovechada para impartir cursos de aprendizaje de manera Online, es así que; el desarrollo del internet ha hecho que las instituciones de educación superior tanto públicas como privadas, utilicen este medio novedoso como recurso y herramienta de aprendizaje, los maestros y profesores de las casa de estudio, han ido aprovechando estos desarrollos tecnológicos para crear páginas web, con el fin de integrar las clases de salón, con las dictadas de manera virtual, esta simbiosis ha significado una mejora de la educación impartida. 
Como se afirmara al principio; las instituciones universitarias han venido sufriendo cambios acelerados, producto de los nuevos desarrollos tecnológicos, ellas se han ido adaptando a esos nuevos cambios, inclusive; algunas se encuentra más adelantadas que otras, este desarrollo, en donde muchas instituciones de educación superior se encuentran por encima de otras en cuanto a su calidad educativa, está trayendo como consecuencia que en su valoración y acreditación los requisitos para aceptar estudiantes de otras instituciones de educación superior, no se encuentren a la par de las exigencias de estas universidades, al respecto; éstos son los retos y oportunidades en lo que están inmersas las instituciones de educación superior, cómo es lograr la unicidad en los tipos de acreditación que requieren algunas de estas universidades o instituciones de educación superior, este reto demanda de una nueva forma o nuevos métodos de acreditación, puesto que, muchas instituciones de educación superior se encuentra rezagadas y las normas de acreditación no son tan exigentes como aquellas que están a la vanguardia de los nuevos desarrollos tecnológicos y por ende, más avanzadas en cuanto a la educación impartida.

En este mismo orden; la tragedia que ha significado el surgimiento el Covid-19, ha afectado en todos los órdenes la vida institucional de la educación pública y privadas a nivel mundial, y como caso paradigmático la acreditación viene a formar parte de esos cambios, que se han visto acelerados, producto de la pandemia, ahora bien; desde hace varios años la acreditación universitaria, son las herramientas fundamentales de la calidad educativa, éstas han ido evolucionando hasta alcanzar estándares internacionales en muchos países, es así; que la mayoría de éstos, cuentan con sistemas de acreditación en desarrollo y evolución constante.

En lo que se refiere a esto, existen varias preguntas ¿Qué es el acreditación? ¿Qué significado tiene hoy en día?¿Cuál es el porvenir de la acreditación? ¿Cuáles son los procesos para el desarrollo de la misma? Al respecto; el fin último de la acreditación es la calidad en educación, sin embargo; no todos los gobiernos y por ende sus instituciones de educación poseen el mismo criterio para la evaluación de las instituciones de educación superior, los organismos encargados de validar la calidad educativa, anteponen sus propios criterios y como tal, decide cómo desarrollarlos y aplicarlos, estableciendo como norte la excelencia educativa, se debe hacer énfasis en lo que establece la real academia de la lengua, en donde fija como concepto, que la acreditación "es un documento que acredita la condición de una persona y su facultad para desempeñar una determinada actividad o cargo", no obstante; la aplicación a una institución de educación superior de las normas de acreditación, exigen criterios muy definidos y estrictos, porque se trata de un ente corporativo, una institución dedicada a la educación de individuos, que van al mercado laboral y de una u otra manera generarán cambios dentro de la sociedad. De allí; la importancia de la acreditación de cualquier institución basada en criterios acorde a las necesidades sociales, la 
acreditación significa calidad, pero; esta calidad debe estar dirigida a aquellas necesidades presentes dentro de la sociedad

Aunado a esto; la crisis generada por el Covid-19, está planteando nuevos retos y oportunidades para aquellos entes dedicados a la acreditación de las instituciones de educación superior, éstos son nuevos paradigmas que se están rompiendo con lo que hasta hace poco se venía haciendo para acreditar a las instituciones educativas superiores, se debe notar; que las premisas fundamentales de la acreditación son la pertinencia, entendida ésta; como aquella por medio del cual se instauran, una variedad de relaciones entre la universidad y el entorno en donde ellas tienen su ámbito de acción y como segunda premisa el compromiso social, explica la UNESCO:

“... La pertinencia es un factor determinante que tiene en cuenta la concordancia entre las
misiones de las instituciones de educación superior y las expectativas de la sociedad. Esto afecta
el papel y lugar de la educación superior en la sociedad, así como; la acreditación como mecanismo
para la garantía del compromiso social de las universidades, como el acceso y la participación, la
educación y el aprendizaje, la función de investigación de las universidades, la responsabilidad de
la educación superior para con otros sectores de la sociedad, el mundo del trabajo y los servicios
prestados a la comunidad..."

Por supuesto; que estos lineamientos establecidos por la UNESCO, están fundados en un contexto, en donde la situación de las instituciones de educación superior en todos los países estuviera estable, no siendo el caso en la actualidad, en donde la crisis generada por el Covid-19, ha significado un parteaguas, por las condiciones de seguridad implantados por todos los países, en donde la pandemia está haciendo estragos. El Covid-19, ha paralizado totalmente la educación, obligando a los discentes a no recibir la educación respectiva, esto ha generado en los estudiantes, dificultades para acceder a la educación producto de muchos factores, tales como; el traslado a las instituciones, mala conectividad en la red, para los que reciben la educación a distancia, más allá de estas dificultades que se le presentan a los estudiantes, se encuentran problemas mucho más profundo, como es: la desigualdad presente entre los estudiantes, lo que coadyuva a la marginación, sobre aquellos estudiantes más desfavorecidos, asimismo; la crisis encontró a una planta profesoral mal preparada para afrontar el reto de una educación virtual.

Hay que hacer notar; que aunque la educación superior viene haciendo adelantos desde que surgió el internet, no es menos cierto; que la pandemia encontró a la academia desprevenida, porque no todo los programas de educación superior se hacen de manera virtual, el mayor porcentaje de la educación superior, se hace de la manera tradicional, la educación a distancia sólo es un complemento de la educación tradicional, es por ello; la obligación de los entes de educación superior dar el salto hacia la educación a distancia, esto trae consecuencias trascendentales, es así: que los organismos encargados de la acreditación de la educación superior, deben replantearse bajo qué parámetros serán acreditados las 
nuevas instituciones que surjan con una educación totalmente virtual, toda esta situación, se ve mejor reflejada en las instituciones superiores de América latina, en donde la educación a distancia, apenas representa un porcentaje mínimo de la educación tradicional, Segrera (2008),en el libros tendencia a la Educación Superior en el mundo y en América latina y el Caribe explica:

\begin{abstract}
“...Los procesos de evaluación y acreditación - que nos brindan mayor información sobre las IES, la masificación de la educación superior, su comercialización, el ataque a la concepción de su condición de bien público, la emergencia de las TIC, el auge de los nuevos proveedores y el deterioro de las condiciones salariales y de trabajo de la profesión académica, entre otros factores, - han llevado a un debate sobre lo que muchos consideran un creciente fenómeno de corrupción académica. Es decir, a la obtención de calificaciones, títulos universitarios, puestos académicos o certificaciones de acreditación en el caso de las IES, mediante procedimientos irregulares tales como: pago de sobornos, venta de títulos sin cursar los estudios adecuados, vender una plaza a un candidato en lugar de otorgarla a quién corresponde, acreditar a una IES o programa sin los requisitos a cambio de pagar cierta cantidad"...
\end{abstract}

De acuerdo con esto; existe un número de circunstancias que son apremiantes y en donde las instituciones de educación superior tienen la última palabra, todos estos factores atentan contra la calidad y la excelencia en la educación superior, es por ello; la importancia de los organismos encargados de la acreditación en las universidades, el autor por supuesto que habla de las universidades en Latinoamérica y el Caribe, debido a su poco desarrollo y avances en los que a las normas de acreditación se refiere, Ahora bien; si se compara; la educación que se imparte en aquellas sociedades más avanzadas desde el punto de vista tecnológico, se puede hacer símil o comparación con aquellos países, en donde el desarrollo tecnológico es menos avanzado, por lo tanto la educación superior allí, aunque es de excelente calidad. En cuanto; al desarrollo de la educación a distancia están menos adelantados, lo que plantea la pregunta de ¿cómo debería ser la acreditación en aquellos países en donde la práctica educativa es de manera tradicional, con un mínimo porcentaje de educación virtual? Al respecto; en estos países es tomada la educación virtual, como un apéndice de la tradicional, pocos países en América latina han avanzado en la educación virtual, como aquellos más industrializados, en donde se ofrece una amplia gama de carreras virtuales, al respecto Zapata-Ros (2015) en "Pensamiento computacional: Una nueva alfabetización digital", dice:

\footnotetext{
"En la actualidad las instituciones y agencias competentes, los expertos y los autores de informes de tendencia se han visto sorprendidos por un hecho: la sociedad y los sistemas de producción, de servicios y de consumo demandan profesionales cualificados en las industrias de la información. Particularmente en el mundo desarrollado se da la paradoja de países y regiones con un alto índice de paro en las que actualmente se quedan sin cubrir puestos de trabajo de ingenieros de software, desarrolladores de aplicaciones, documentalistas digitales, por falta de egresados de las escuelas técnicas, por falta de demanda de estos estudios por parte de potenciales alumnos y sobre todo por la falta de personal capacitado. Ante esta situación los sistemas educativos de los países más sensibles han abordado el problema desde la perspectiva de una reorganización del curriculum en la mayor parte de los casos donde se ha producido esa reacción. Sin embargo la cuestión de
} 
fondo supone la aparición de unas nuevas destrezas básicas. Las sociedades más conscientes han visto que se trata de una nueva alfabetización, una nueva alfabetización digital, y que por tanto hay que comenzar desde las primeras etapas del desarrollo individual, al igual como sucede con otras habilidades clave: la lectura, la escritura y las habilidades matemáticas, e incluso estudiando las concomitancias y coincidencias de esta nueva alfabetización con estas competencias claves tradicionales".

Esto quiere decir una cosa, el mundo hoy en día, está cada vez más interconectado a través de las herramientas tecnológicas, lo que hace más exigente los programas educativos que se imparten en las instituciones de educación superior, es por ello; que el desarrollo acelerado de las TIC, ha hecho que más educadores encuentren en la web, una herramienta de soporte a la educación tradicional y han visto ésta, como una alternativa a la enseñanza, esto ha generado conceptos tales como "E-learning" que es como se llamaría el aprendizaje electrónico, todo ello a través de videoconferencia y cursos interactivos, otro concepto que se manejan actualmente, es el de B-learning, que no es más que el aprendizaje semi presencial. Este tipo de desarrollo en educación interactiva, esta nueva manera de aprendizaje virtual llamadas E-learning, puede ser A-Sincrónico y Sincrónico, el primero se puede describir como aquella modalidad de aprendizaje en donde el docente y el discente, interactúan en tiempos o momentos distintos, así como espacios diferentes, el docente introduce todo el material, y la documentación y toda las actividades a través de la red, lo que le permite al discente o a los alumnos conectados a la red planificar la manera de estudio, no hace falta la conexión directa con el docente. Igualmente; dentro de las herramientas más utilizadas para la educación Sincrónicas, se encuentra el correo electrónico, el Chat, el MSN, foros, así como la pizarra informativa, de esta manera los alumnos son autónomos y capaces de desarrollar su propio aprendizaje.

Ahora bien; en el E-learning Sincrónico, el docente y el dicente interactúan a través de la red, no importando la distancia, ni el espacio en que se encuentre, se pueden escuchar, leer y ver en un mismo momento, este tipo de educación virtual que es en tiempo real, como si fuera una clase presencial, la diferencia es que no se encuentran físicamente presente, todo ello es virtual a través de salas de chat, videoconferencias, etc. Sin embargo; la efectividad de esta nueva modalidad educativa podrá no ser positiva, sino está fundamentada en un modelo pedagógico, sin pedagogía, el uso de la tecnología no alcanzaría su fin que es la optimización del aprendizaje.

De acuerdo a este planteamiento; la universidades se encuentran inmersas en una transformación producto de los nuevos desarrollos tecnológicos, que han invadido la cotidianidad humana, estos nuevos cambios se han visto acelerados por la llegada del Covid-19, es allí; en donde los nuevos retos y oportunidades, son tomados en cuenta para lograr el fin último de estas, que es una educación con calidad y excelencia, dentro de estas variables, es la acreditación uno de los factores más importantes a tomar en consideración para lograr este cometido, ahora bien; que parámetros son los más adecuados para saber si 
una institución de educación superior está a la vanguardia de la calidad y excelencia educativa, visto; los nuevos avances tecnológicos en donde cada día se hace más latente la educación a distancia y por otra parte; cual es la forma de regular la actividad educativa, para que cada día estén más acorde a las necesidades que exige la sociedad actual y donde la acreditación es clave, explica Kuhn (2020)

\begin{abstract}
"Las revoluciones científicas se producen cuando las viejas teorías y métodos ya no pueden resolver los nuevos problemas. A estos cambios de teorías y de métodos los llama "cambios de paradigma". Existe una preocupación generalizada de que las experiencias educativas que se proveen actualmente en muchas escuelas no serán suficientes para formar alumnos capaces de afrontar el futuro (UNESCO, 2004). Las TIC, la sociedad de la información han creado un nuevo paradigma educativo centrado en el conocimiento. Se hace obligatorio que las personas desarrollen la competencia de aprender a aprender, ya que los conocimientos que se obtienen al terminar una profesión están cambiando y esto obliga a la actualización permanente ya sea de manera formal o informal a través de redes de aprendizaje, de ambientes virtuales de aprendizaje, de cursos virtuales, presenciales o semipresenciales. Claramente se pueden identificar dos paradigmas en la educación. Un paradigma tradicional en donde el profesor es el centro del proceso educativo, el cual es poseedor de los conocimientos y los transmite a los estudiantes que los recibe normalmente en un aula de clases, el estudiante es un receptor y reproductor del conocimiento. Este paradigma se corresponde con la sociedad industrial, en el cual se requería que los profesionales aplicaran lo que aprendían en la universidad, es una época en donde el ciclo de vida de los conocimientos se podía medir por décadas.
\end{abstract}

De acuerdo a este planteamiento; las instituciones de educación superior tienen ante sí, problemas que deben resolver, son cambios revolucionarios que la obliga a estar más acordes a las necesidades, de una sociedad que cada día es más exigente y entre estos cambios está el tránsito de una educación netamente tradicional a una educación más moderna como es la educación virtual, la cual está generando el rompimiento de un paradigma, por las actuales exigencias de la sociedad, a la cual ya no es posible darle respuesta a través de los métodos tradicionales de educación, por el alto crecimiento y demanda estudiantil.

Al respecto; el desarrollo de las TIC, han traído el surgimiento de una cantidad de novísimas carreras, todas ellas dedicadas a los nuevo desarrollo tecnológico, las instituciones de educación superior ha ido dando respuestas a este nuevo tipo de demanda de carreras nuevas, sin embargo muchas veces han sido desbordadas, lo que ha traído aparejados el surgimiento de universidades privadas de todo tipo, para tratar de paliar la demanda estudiantil, pero surge otro tipo de problemas que es la calidad y la excelencia educativa, es allí; en donde tiene prevalencia y es fundamental la acreditación que no es más, que la valoración a través de procedimiento y de normas estrictas la calidad educativa.

Es de hacer notar; que la acreditación en las instituciones de educación superior, es una forma de control de calidad, dirigida fundamentalmente a que las mismas cumplan una serie de requisitos, para que luego de pasado dicho examen, los resultados sean presentados a la sociedad, que son al final de cuenta, los que a través de los impuestos soportan el mantenimiento de dichas instituciones, tanto públicas como 
privadas, las primeras que subsisten a través de los recursos del estado y la segunda a través del cobro de aranceles, tanto unas como otras, son reguladas a través de leyes, para que sean capaces de transformar su entorno, y darle respuesta a las demandas sociales.

Como se afirmó al principio; el fin último de las instituciones de educación superior es la calidad y excelencia educativa, para ello; la acreditación es un requisito "prima facie" para ser reconocida como una institución de primer orden, en América latina y el Caribe por lo general la acreditación corresponde al estado, esto conlleva a una enorme presión, primero, por la crisis económica que afronta el estado en general, lo que origina una reducción presupuestaria significativa en la educación pública, aunado; al acelerado crecimiento de la educación privada, también; el surgimiento de otros tipos de enseñanza, al respecto (DIDRIKSSON, 2010) dice:

\begin{abstract}
En América Latina y el Caribe, varios factores han supuesto un gran desafío para el mantenimiento de la calidad, entre ellos: la enorme expansión de la matriculación en un período de crisis económica, la reducción de la partida presupuestaria estatal para educación superior, y la aparición del sector privado y de diferentes modelos de educación superior
\end{abstract}

A decir; del autor son muchos los factores que afectan directa e indirectamente la calidad de la educación superior hoy en día, aunque las instituciones de educación universitaria, hacen grandes esfuerzos por ponerse al día y estar a la vanguardia de los nuevos retos y oportunidades, que han traído los nuevos desarrollos tecnológicos, no es menos cierta, que los presupuestos dedicados a tal fin, cada días son más exiguos, vista la crisis económica por la que pasan sus respectivos países. y como corolario a todas estas crisis y donde la universidad coloca todos sus esfuerzos para lograr salir adelante y dar una mejora calidad educativa a la sociedad, surge un escollo en el camino, como es el Covid-19, esta crisis ha retrasado los planes estratégicos de las instituciones de educación superior, pero también ha hecho de éstas más audaces en la toma de decisiones, lo que ha configurado una nueva forma de educar, es así; que las instituciones de educación superior han colocado todo su empeño y recursos humanos para no paralizar las clases, haciendo uso de las TIC, como una de las herramientas tecnológicas más novedosas para la educación a distancia.

Se puede inferir; que la crisis generada por el Covid-19 ha hecho de las instituciones encargadas de la acreditación universitaria, repensar la forma en cómo se venían realizando éstas y es que los entes de educación superior se están enfrentando a retos jamás pensados, el vertiginoso y exponencial crecimiento de la pandemia, obligó a cerrar todas las instituciones universitarias con consecuencias, tales como; la obligación de los estudiantes a quedarse en sus casas, dejando todas las estructuras educativas de los países prácticamente vacías, no cabe duda; que las decisiones tomadas por los entes encargado de la educación superior, fueron tomadas de manera acertada, lo que ha ayudado a repensar la universidad 
tal como se conocía, son retos y oportunidades y es lo que se llamaría una reinvención de todo el sistema educativo, las decisiones tomadas de manera audaz, para salvar el año académico en curso, son signos de cambios, no sólo coyunturales, sino; también de cambios estructurales, la rapidez de respuesta asertiva a lo que sucedía ha significado todo reto y una oportunidad para dar el salto hacia un nuevo tipo de educación superior.

En lo concerniente; a este nuevo paradigma que se le presenta a la educación superior, ya estos cambios se venía gestando desde la llegada de la Web, éstas venían tiempo hace, desarrollando y adaptándose a estos nuevos cambios, la crisis del Covid-19, no ha hecho más que acelerar los cambios, sin embargo a estos nuevos retos y oportunidades presentes, se les suman otros de primerísimo orden, como es la calidad y excelencia educativa, lo que avizora una serie de obstáculos en cuanto a la acreditación de estas instituciones de educación superior, todo ello genera una suerte de incertidumbre, dentro de estas, a todo ello; la que tiene mayor preeminencia, es el cuestionamiento a las condiciones de calidad educativa, todo esto tiene que ver con el uso de las TIC (tecnologías de la información y las comunicaciones) para la educación a distancia y su posterior desarrollo.

En lo tocante; a la acreditación de las instituciones de educación superior ya algunos países que cuentan con los entes encargados de la acreditación, se encuentran estudiando la forma y los nuevos métodos, de cómo serán los nuevos modelos a aplicar para certificar a las instituciones de educación superior que lo requieran, mientras los entes que se encargan de esta labor en muchos países todavía no han asimilado los cambios que se están generando de manera acelerada, sin embargo; las universidades llevan la delantera y están afrontando estos retos y oportunidades de manera práctica y eficiente, teniendo siempre como norte, la calidad y la excelencia educativa. Son notables las experiencias de muchas universidades que al poco tiempo de tomar la decisión de cerrar sus puertas, a la semana siguiente, habían resuelto la forma de seguir dando clases a sus alumnos de manera virtual utilizando las TIC, y todas aquellas plataformas tecnológicas usadas para este fin.

Es importante señalar; que las universidades son las primeras instituciones de educación superior, en dar respuesta de manera eficaz y eficiente, éstas lideraron en un tiempo perentorio la habilitación de profesores y estudiantes en las plataformas virtuales como Moodle, así como; el acceso a aquellas herramientas para los encuentros "sincrónico" con los estudiantes, asimismo; se ha hecho uso corriente de las otras tecnologías tales como: WhatsApp, Classronn, Zoom, como contrapartida; a todas estas iniciativas lideradas por las autoridades de estas instituciones de educación superior, también surgieron nuevos retos de cómo hacerle llegar la educación virtual, a aquellos estudiantes que no contaban con los artilugios tecnológicos necesarios para poder recibir las clases correspondiente, debido a su situación 
económica. En otras palabras; la mayoría de instituciones de educación superior, tienen ante sí un reto insoslayable que no es otro que mejorar las condiciones de calidad y de excelencia educativa.

En todo caso; los retos y oportunidades planteadas a las instituciones de educación superior vienen aparejados con la calidad y la excelencia, además de la eficacia y la eficiencia en la toma de decisiones para lograr su acreditación, hay que agregar; que las universidades hoy en día, se están enfrentando al reto de la globalización trayendo como consecuencia retos y oportunidades, pero a su vez; desafíos como nunca antes, en donde se definirá el futuro de la educación superior.

Es importante decir; que desde el origen de las universidades, estas; han tenido como valores fundamentales, la libertad de cátedra, la autonomía, la investigación, la evaluación, todos estos valores vigentes desde la fundación de las instituciones de educación superior, hoy en día tienen más validez, sin embargo; dentro del contexto actual, la globalización está haciendo que estos pilares en donde se fundamenta la educación superior, corran peligro, se debe hacer énfasis, en que la globalización es un fenómeno permanente y en constante evolución, que está haciendo de estos valores menos importantes dentro de la educación superior

Es fundamental; tener presente que la educación superior tiene entre sus metas esenciales, la creación de conocimiento para hacer una sociedad más justa y equitativa, estos valores están en constante contradicción debido a la globalización, cada día son más las instituciones de educación universitaria de cortes privada, en donde el ánimo de lucro priva por sobre la calidad y la excelencia educativa, son todos estos problemas los que deberá resolver la educación superior y los organismos encargados de la acreditación de estas nuevas instituciones de educación universitaria, éstas premisas, son necesarias para poder dar respuestas y así contribuir a las necesarias transformaciones de la educación superior, otro punto a destacar; son los constantes reajustes en las inversiones públicas dedicada a la educación superior, trayendo como consecuencia una baja calidad y excelencia educativa, son problemas a lo que deberán dar respuesta los organismos o instituciones dedicadas a la labor de acreditación de las instituciones de educación superior para propender a la calidad educativa. Es así que; la UNESCO (1998) en una conferencia sobre educación superior establecía:

"La Conferencia Mundial sobre la Educación Superior" (1998), convocada por la UNESCO, y en las Comisiones de Seguimiento de dicha Conferencia, se hicieron sugerencias valiosas de cómo afrontar los desafíos más urgentes. Como por ejemplo: la actualización permanente de los profesores, de los contenidos y del currículo; la introducción de redes electrónicas para el aprendizaje; traducción y adaptación de las principales contribuciones científicas; modernización de los sistemas de gestión y dirección; e integración y complementación de la educación pública y privada así como de la educación formal, informal y a distancia. 
Al respecto; en dicho documento de la conferencia mundial sobre la educación superior del año 1998, ya las universidades venían trabajando sobre el desarrollo de las nuevas tecnologías y algo muy importante la integración tanto de la universidad pública como privada, así como; las distintas formas de educación existente y como algo novedoso para esa época "la educación a distancia", puede señalarse; la importancia para las instituciones de educación superior, el estar a la vanguardia de los desafíos presentes y futuros, son problemas que plantean una discusión más profunda en los momentos actuales, porque como se ha reiterado en varias oportunidades durante la disertación de este escrito, los momento que está viviendo y causando la crisis de Covid-19,dentro de toda la sociedad.

\section{MATERIAL Y MÉTODOS}

Ahora bien; en el presente ensayo Covid-19: Retos y Oportunidades en el Proceso de Acreditación de las Universidades, existe una variedad de informes y trabajos con el propósito de dar respuesta a los nuevos avatares que se están presentando dentro de la educación superior, en donde la crisis generada por el Covid-19, ha causado un terremoto que ha causado que los cimientos de las instituciones de educación superior sufran cambio como nunca antes había pasado, pero que a su vez; ha permitido a las instituciones que acreditan a las universidades, plantearse retos y oportunidades, esto tiene mucho que ver en cómo las universidades se están reinventando, esto es; una nueva forma de educar y la mejor solución y más novedosa fue poner a punto y acelerar la puesta en marcha de la educación a distancia, en otras palabras; todo el conocimiento acumulado desde que el internet hizo su aparición, hace varias décadas atrás, ha servido como soporte a la nueva forma de educar. Todos estos cambios están produciendo en las instituciones encargadas de acreditar a dichas universidades, proponerse nuevos métodos para la acreditación, esto se traduce en nuevas reglas y normativas.

De esto se desprende; que la acreditación a las instituciones de educación superior hasta hace poco tiempo, eran acreditadas de la manera tradicional, puesto que; la educación hasta la aparición del coronavirus (Covid-19) era practicada de manera tradicional, pero la hecatombe producida por la pandemia obligó al cierre total de toda las instituciones universitarias, ¿Qué significa esto? Simplemente; que los estudiantes ya no están asistiendo a las universidades y están recibiendo clases a través de la red, lo que genera un sinnúmero de preguntas, igualmente de respuestas que deben ser sometidas a consideración. Dentro de este marco; las preguntas que en este momento se hacen los organismos encargados de la acreditación de las instituciones de educación superior y la sociedad en general son: ¿Qué parámetros se deben establecer para medir la excelencia académica, en tiempos de educación virtual 
producto de la crisis generada por el Covid-19? ¿Cuál será el método utilizado para la acreditación institucional Universitaria, en una educación a distancia?

En líneas generales; los organismos de acreditación tienen ante sí un arduo trabajo para proponer y para dar respuestas a todas estas preguntas, que en este momento se hacen las instituciones universitarias, los estudiantes, los docentes universitarios, igualmente la sociedad en general. Se hace necesario resaltar; que los organismos de acreditación se encuentra ante un desafío, puesto que la educación está pasando de una educación directa a una educación totalmente mediatizada, esto es; en donde la utilización de las nuevas tecnologías tales como, ordenadores y otros aditamentos tecnológicos para dictar las clases a través de audio-conferencias, videoconferencias, chat etc. Es por ello; que los organismos encargado de la acreditación deberán entender, que se está generando un cambio estructural desde una educación tradicionalmente dictada en cuatro paredes, lo que los expertos llaman educación multisensorial, en donde el estudiante está en contacto directo con el docente donde lo puede oír, escuchar, palpar oler, sentir; a una educación limitada, entendida ésta como aquella educación en donde el docente y el discente, no tienen contacto directo, puesto que se restringe a dos sentidos: la vista y el oído. Pierre (1999) en su libro ¿Qué es lo virtual?

\footnotetext{
“...lo virtual conjetura una reconversión de las coordenadas clásicas de espacio y tiempo por un sistema donde las mismas, o han desaparecido, o más bien, se han virtualizada. Por ejemplo, en la educación, la estructura espacial clásica del aula se "vaporiza" para entrar en una especie de aula simulada e ilimitada, pasando el espacio a ser una realidad de simulación, virtual. Además, se rompe la estructura del tiempo; un curso deja de impartirse en un período, horario determinados; el proceso de estudio y consulta de los materiales se relativiza, como la tutoría por parte del profesor o el trabajo en grupo con otros compañeros; en la realidad virtual se realiza fuera del marco temporal, en un sistema donde el tiempo se ha virtualizada adaptándose a nuestras demandas de flexibilidad y accesibilidad..."
}

Al respecto; el autor de forma simbólica establece un símil o comparación, en donde las estructuras diseñadas para albergar una gran cantidad de estudiantes, en este caso las universidades, quedan relegadas en un segundo plano. Haciendo una abstracción de lo dicho por Pierre en su libro ¿Qué es lo virtual? Los organismos encargados de la acreditación en la educación superior, se les presentan nuevos retos, pues tendrán que definir de qué manera acreditar las instituciones universitarias totalmente virtuales, en donde la presencia física de los estudiantes en las aulas de clase ya no será necesaria.

Aunado a esta situación, los organismos o entes encargados de dar las acreditaciones necesarias para estas nuevas instituciones de educación superior virtualizadas, se le suma el hecho de las condiciones tecnológicas presentes en los países de bajos recursos, cuya capacidad científico-técnica no le permiten estar al día con los nuevos adelantos y desarrollos tecnológicos en materia de educación virtual o a distancia, lo que no les habilitaría para cumplir con los requisitos necesarios y las garantías para proveer de calidad y excelencia educativa, como imperativo "sine qua non" para la acreditación. 
Si bien es cierto; los avances en las nuevas tecnologías de la información dedicadas a la educación a distancia y donde las universidades e instituciones de educación superior son las que más han aportado a la nueva realidad planteada, originados por la crisis del Covid-19, los organismos de acreditación se han propuestos una serie de objetivos, entre estos tenemos la creación de agencias de acreditación, dedicadas exclusivamente a dar seguimiento a las instituciones de educación superior, como paso previo para su acreditación, sin embargo; como se ha reiterado, la crisis generada por la pandemia y las respuestas dadas de manera rápida y efectiva por las universidades, al proponer de manera inmediata la educación virtual con los recursos tecnológicos disponibles, para no cerrar la academia y por ende la educación, ha supuesto; también serios retos a los organismos de acreditación, se debe tomar en cuenta que no son los mismos parámetros establecidos para acreditar a una institución de educación superior tradicional, a una universidad dedicada a dictar clases de manera virtual o a distancia.

Cabe considerar; que la creación de agencias dedicadas a la acreditación de las instituciones de educación superior, se viene dando de manera paulatina en muchos países de América latina con universidades prestigiosas, los objetivos de estas agencias es lograr la calidad y la excelencia educativa tanto en sus programas, como en sus instituciones de educación superior, otro motivo de estas agencias de acreditación es controlar la proliferación de instituciones de educación superior con fines de lucro. En este mismo contexto; este estudio del Covid-19: Retos y Oportunidades en el Proceso de Acreditación de las Universidades, los entes encargados de esta tarea deberán plantearse nuevos métodos, nuevas reglas, nuevas normas.

En otras palabras el Covid-19 así como está obligando a las instituciones de educación superior a reinventarse, igualmente los organismos de acreditación deberán tomar en cuenta todos estos cambios. Y entre los más importantes, se encuentra el cambio de una educación tradicional, dictada dentro de cuatro paredes, en estructuras de concreto en donde se albergan miles de estudiantes, a una educación virtual, en donde ya esta estructura no tiene razón de ser, simplemente quedarán como resguardo de los servidores encargado de mantener abierta las aulas virtuales. Estas vetustas y obsoletas edificaciones, sólo servirán en un futuro cercano para mantener el personal mínimo necesario para el mantenimiento del equipo tecnológico, servidores, computadoras etc. Dedicadas exclusivamente 24 horas del día, para las clases virtuales.

En esta perspectiva, tanto el personal docente como el estudiantil, permanecerán en sus casas y desde allí cada uno hará lo propio, de hecho; este fenómeno se está viviendo en este momento, en dónde; las universidades están totalmente cerradas, con un personal mínimo necesario para labores administrativas y los profesores utilizando las herramientas tecnológicas para vaciar las clases a través de la red, igualmente los estudiantes recibiendo las clases de manera "Asincrónica" y en otros casos de 
manera "Sincrónica", dentro de este marco; los organismos de acreditación para las instituciones de educación superior, deberán tomar en cuenta las situaciones planteada y formarse nuevos criterios para la evaluación y acreditación de estas universidades y así lograr su cometido final, que es la calidad y la excelencia educativa.

En torno a esto; los procesos de acreditación dirigido por los organismos encargados de esta, tienen ante sí cuestiones a resolver, como por ejemplo: que población estudiantil recibe educación a distancia de las universidades que, en este momento están embarcadas en dicho proyecto, vista la situación y crisis generadas por el Covid-19, son datos relevantes, para la toma de decisiones y acreditación de estas instituciones de educación superior, se debe tomar en cuenta quienes tienen los medios suficientes para recibir este tipo de educación, todo esto viene a colación porque en el informe de la UNESCO (2015), sobre la ciencia hacia el año 2030, explicaba dicho informe:

\begin{abstract}
“en el 2008 el 23.13\% de la población mundial tenía acceso a internet, mientras en el 2014 alcanzaba el 37,97\%. Este crecimiento en la accesibilidad variaba según la región. En América Latina se había pasado del $27.09 \%$ al 47,59, es decir, casi uno de cada dos habitantes tenía acceso. Sin embargo, cuando observamos quiénes acceden se develan las enormes inequidades existentes en las sociedades. En su mayoría el acceso es urbano contra un casi nulo acceso en el campo, pero en las ciudades es la clase media y los profesionales quienes tienen mayor conectividad. La ciudad tiene periferias, barrios que reflejan toda la exclusión, incluido el acceso al internet. A eso se le suma el tipo de conexión a la que se accede y sus posibilidades de uso.
\end{abstract}

En este informe; presentado por la UNESCO se establece una idea general del acceso a internet de la población en América latina, estos datos reflejan de manera fehaciente el grado de conectividad de una población dada, estas son variables a tomar en cuenta por las agencias u organismos encargados de dar acreditación a aquellas instituciones de educación superior de manera virtual,que como se dijo anteriormente, la calidad y la excelencia educativa son su fin último. Otro dato importante a tomar en cuenta por los organismos de acreditación para las instituciones de educación superior es el informe presentado por Global Digital (2019)

\begin{abstract}
..."Señalaba que el 57\% de la población mundial tenía acceso a internet en sus distintas modalidades; el $43 \%$ de rezago es el dato más relevante, nuevamente la exclusión centrada en el campo y las zonas populares de las ciudades. El estudio de Global Digital muestran la importancia que le dan los usuarios al uso del internet. Respondiendo en el estudio citado a varias alternativas, los consultados nos muestran que se usa internet en un $92 \%$ para ver videos, 58\% para la televisión en streaming, 30\% para juegos en línea, 23\% para ver jugar a otros y un $16 \%$ para ver partidos o Sports...."
\end{abstract}

En el presente informe se puede detallar de manera gráfica que, no existe una cultura del uso educativo del internet, un dato importantísimo, si se quiere, fundamental a la hora de acreditar a cualquier universidad en modalidad virtual. Al respecto; en este estudio,Covid-19: Retos y Oportunidades en el 
Proceso de Acreditación de las Universidades, todos los datos presentados son de suma importancia para la creación de una nueva normativa dirigida a lograr la excelencia educativa, porque; como se ha visto en los actuales momentos, las instituciones de educación superior tradicionales se aprestan a dar el salto hacia una educación totalmente virtual, este paso será trascendental, para el devenir histórico de la sociedad, su significancia y magnitud se pierden de vista, pero; si este cambio histórico de una educación tradicional a una virtual, no va acompañado de aquellos organismo encargado de normar las acreditaciones respectivas para lograr la calidad y excelencia educativa, será un salto al vacío, porque; sólo la acreditación permitirá una educación de calidad justa y equitativa dirigida a la mayoría de la población que cada día es más exigente.

Continuando con el tema en cuestión; el tema de la acreditación en las instituciones de educación superior, ha sido debatido desde hace décadas, siempre ha estado como asunto prioritario en las agendas de los diferentes países que cuentan con agencias dedicadas a esta labor. De hecho; siempre han tenido mucha profusión y debate en los círculos de expertos en acreditación, éstos han intentado conceptuar que significa la calidad en educación, la significación para dar respuesta a este tipo de preguntas, adquiere mayor relevancia, ahora que las instituciones universitarias se encuentran inmersas en la crisis generada por el Covid-19, por supuesto; que ésta pandemia les está dando réditos, a dichas instituciones, de qué manera? Pues las ha obligado a reinventarse a sí misma, es por ello; que cuando se habla de crisis universitaria, en realidad también se puede hablar de retos y oportunidades.

En cuanto a la acreditación; ésta va de la mano con aquellos desarrollos y propuestas universitarias en cuanto a la calidad y excelencia, atendiendo estas consideraciones; para lograr este cometido los entes dedicados a esta labor, toman en cuenta las necesidades sociales presentes en un país, todas ellas deberán estar en correspondencia con las directrices y planes de estudio de las instituciones de educación universitaria, el sentido de esta correspondencia tiene que vercon las exigencias de la sociedad como un todo, se puede inferir; que la evaluación de la educación superior por parte de los órganos de acreditación, es lograr la continuidad de la calidad y la excelencia en estos institutos de educación universitaria.

Considerando que; para lograr la calidad y la excelencia en la educación superior, hubo la necesidad de crear entes externos cuyo fin último, era hacerle seguimiento a los procesos internos para examinar los procesos educativos llevados a cabo por éstos entes de educación superior, con el tiempo se ha demostrado que la autoevaluación llevadas a cabo por la misma universidad, tiende a mejorar la calidad y la excelencia educativa, puesto que; es la institución universitaria la que mejor conoce sus procesos internos y su entorno social.

Si bien es cierto; que la autoevaluación ha sido fundamental en el devenir histórico-social dentro de la universidades, no es menos cierto que la globalización y el desarrollo científico-técnico de los países 
del orbe, obligaron a la mayoría de los países a la creación de organismo encargados de la evaluación académica, todo ello; por la necesidad de homologar los contenidos educativos, así como; la gran movilidad social de estudiantes entre los diferentes países, todo ello ha configurado; una serie de agencias o instituciones encargadas de dar las acreditaciones necesarias, a aquellas instituciones superiores, luego de pasar por una serie de normas y exámenes rigurosos, todas ellas con el fin certificar la calidad y excelencia académica. Hay que hacer notar; que todo los proceso de acreditación externa en las instituciones de educación superior, lleva intrínseco el perfeccionamiento de la calidad y excelencia educativa dirigidas especialmente a la sociedad, así como; para ella como institución de educación superior. Es así; que la acreditación como mecanismo de evaluación, le otorga a las universidades esas características que la hacen ver de mucha valía ante la sociedad a la cual sirven y como una institución seria y creíble.

\section{RESULTADOS}

Cabe considerar por otra parte; que en este estudio, Covid-19: Retos y Oportunidades en el Proceso de Acreditación de las Universidades, este proceso, por ahora deberá afrontar retos tales como, la ultra-especialización, crisis en los presupuesto dirigidos a las universidades publicas debido a la paralización del estado producto de la pandemia, la precarización del trabajo docente, no todos elaboran a dedicación exclusiva, un alto porcentaje lo hace por horas o por contrato temporales, pero a su vez; las crisis del Covid-19, ha originado oportunidades para todos los involucrado en el proceso educativo, se está produciendo una verdadera revolución en las instituciones de educación superior, y es el uso de la telemática como recurso de primerísimo orden y fundamental en el nuevo proceso educativo, este nuevo aditamento tecnológico, la telemática, ha sido una de las más novedosas propuesta de seguridad para evitar el contagio producido por Covid-19, en este orden, otro reto a tomar en cuenta es por cuanto tiempo y hasta qué punto las instituciones de educación superior van a mantener la educación virtual, visto que ya varios países han decidido mantener este formato de educación a distancia hasta el próximo año, faltara ver hasta dónde estarán dispuestas a llegar las instituciones de educación superior, con el uso de la telemática para la educación virtual, si éstas serán capaces de dar el salto final hacia una educación totalmente digitalizada, con los consiguientes desafíos de toda índole que esto generara en la sociedad.

Ahora bien; en cuanto a la acreditación en las instituciones de educación superior totalmente virtualizadas, vienen una serie de cuestionamientos, tales como; que rol van a jugar a partir de ahora estos organismos, estos entes, estos especialistas, dedicados a dar la acreditación necesaria a aquellas universidades, que cumplan con los requisitos para recibir el certificado de calidad y excelencia educativa, en un ambiente en donde ya las estructuras tal como se conocen, no existen, donde solo habrá un puñado 
de individuos dedicados a dar mantenimiento a los equipos telemáticos y donde los profesores solo se trasladaran al ente, a recibir tal o cual información relevante de tipo administrativo. Los estudiantes por otra parte solo se trasladaran a estos recintos a recibir los títulos luego de haberse graduado, sin embargo; en este momento en plena pandemia, ya los actos de grado multitudinarios, quedaron totalmente prohibidos, por medidas de seguridad y sanidad producto del Covid-19.

Todo este panorama; constituye un compendio de retos y oportunidades, en dónde; los organismos de acreditación tendrán, sino la última palabra, por lo menos su relevancia será de vital importancia, por la educación mediatizada, y su total digilitacizacion, en los nuevos proceso de acreditación. Estos organismos están en la obligación de adaptarse, de reinventarse, no tienen opción, porque de ellos dependen la calidad y la excelencia educativa, por todos en sabido; la amplia masificación de las TIC, lo que ha contribuido de cierta manera a la evolución de la enseñanza-aprendizaje, irradiándose ésta, a amplios sectores de la población, lo que obliga a los organismos de acreditación a ser más riguroso, a plantearse, nuevas formas de medir la calidad educativa, algunos estudiosos han establecidos que la educación a distancia es solo un complemento de la educación tradicional, al respecto,Viegas, García, Kimura, Scavone, Fronti (2001); en la investigación "Educación Virtual como Complemento de la Educación Formal y Continuada" dijeron:

\footnotetext{
“...En general, en estas instituciones que utilizan la modalidad a distancia, los alumnos estudian en forma independiente pero concurren a los centros regionales para las tutorías. El capacitador es un docente que guía al alumno, que soluciona sus dudas y que generalmente tiene un encuentro presencial. Podemos decir que en la década 1991-2000 algunas de estas universidades abiertas, y otras tradicionales en forma complementaria, se acercan paulatinamente a la enseñanza virtual El alumno puede utilizar material escrito, pero en algunos casos puede acceder a los estudios a distancia, desde su hogar u oficina solamente con una clave de ingreso al sistema de computación; la presentación de la asignatura; el "recibimiento" del tutor (por Internet), el acceso al material y la bibliografía la recibirá interactuando con su equipo de computación..."
}

$\mathrm{Al}$ respecto; establecen estos autores en su investigación, que la educación tradicional, se hace, de las herramientas de la telemática para complementar los estudios de manera formal, para estos investigadores la educación solo se limita a ser un apéndice de la educación tradicional, pero por otra parte, dejan a entrever estos autores, que la educación tradicional se va deslizando gradualmente hacia una educación totalmente virtualizada. A Nunca se esperaban los investigadores, el vuelco desde el punto de vista educativo, que iba a causar el Covid-19, ni la importancia de este cambio tan radical y de una magnitud nunca antes vista, esto; se ve reflejada en los actuales momentos al comprobarse cómo, aquellas Universidades tradiciones con una trayectoria de décadas, debido a la crisis generada por el Covid-19, están migrando o se aprestan a migrar de manera acelerada a una educación totalmente virtual, todo este cambio se venía gestando desde hace décadas, la llegada del Covid-19 lo que ha hecho es acelerar los 
cambios, al respecto; entre las bondades del uso de las nuevas tecnologías de la información y las comunicaciones es que ahora, es posible llegar a poblaciones de lugares remotos, que antes estaban vedados en el uso de la educación virtual por diferentes motivos.

Por consiguiente; son muchos los beneficios, además de los retos y oportunidades que ha traído la pandemia, se puede decir; que la ampliación de las ofertas académicas de manera virtual, está masificando el conocimiento de una manera libre y colocando de manera trascendente, el conocimiento científico, humanístico, técnico y artístico, es allí donde los organismos encargado de la acreditación académica, juegan un rol de primerísimo orden, puesto que son ellos los que le dan el visto bueno a aquellas instituciones de educación superior, luego de los numerosos y rigurosos trámites exigidos, para que éstas logren el objetivo primordial, que es la calidad y excelencia educativa.

Por otra parte; con la crisis del Covid-19, se está configurando una suerte de comunidad educativa totalmente virtualizada, en dónde; docentes, estudiantes, personal técnico-admirativo y obreros, ya no están socializando como hasta hace poco, debido al distanciamiento social y físico, obligado por las medidas de seguridad sanitarias implementadas, esta individualización en el entorno universitario.A decir, de los expertos para la creación de conocimiento se necesita de la cercanía grupal, de la interacción colectiva, es así; que el proceso educativo y por ende del acto que genera conocimiento, está intrínseco ligado a la sociedad como un todo, no es construcción ni formación de manera aislada. Son éstos los retos y problemas a resolver por la academia y los organismos encargados de acreditar a los entes universitarios, debido a que la socialización entre los seres humanos es uno de los valores antropológicos más importante y en lo que se refiere a la educación es esencial en la simbiosis docente-discente y entre discente-discente, ésta relación, entre los actores principales en la educación superior, están por encima de lo estrictamente educativo, ella trasciende y genera normas de convivencia.

\section{DISCUSIÓN Y CONCLUSIONES}

En resumen; la crisis generada por el Covid-19: Retos y Oportunidades en el Proceso de Acreditación de las Universidades, ha sido uno de los acontecimientos históricos que está generando toda una serie de cambios en los cimientos dentro de la sociedad, que traerán consecuencias que afectarán la forma en como concebimos la vida hoy en día y es; la total virtualización de todo lo que se hace en la vida diaria, para ejemplarizar, hoy la mayoría de las cosas se realizan a través de la internet, ésta es una verdad que no se puede eludir y la transformación más trascendental y que cambiara para siempre, en cómo se percibe el mundo actual de las cosas, tendrá como actor y eje principal "la educación".

Sin duda alguna; es la educación la base fundamental de donde se está originando todo este cambio histórico, sin ella no fuera posible todos estas trasformaciones, ella; es el sendero y es y ha sido la guía 
de toda la evolución que se está sucediendo en el mundo. Ahora bien; el paso que está dando la educación tradicional, a una educación totalmente virtualizada, no pudiera realizarse sin la revolución de las TIC, que es origen y germen de la investigación científico-técnica de la educación, sin la educación no hubiese podido ser posible esta revolución tecnológica y ésta; es la que ahora lleva la batuta de éstas trasformaciones. En este aspecto; la puesta en marcha de la educación a distancia, ha invadido todas las áreas de la cotidianidad universitaria, desde la aparición del Covid-19 las instituciones de educación universitaria pusieron en marcha, todos los mecanismos para iniciar la virtualización de la educación.

Esto quiere decir; que están trabajando solo un puñado de expertos encargados de la automatización y mantenimiento de los equipos telemáticos, para dar continuidad a la educación, ya no de manera tradicional, sino a través de las redes, ya no es necesario que los docentes y los discentes, se trasladen a las instalaciones universitarias. Lo que antes parecía imposible, es ahora una realidad, los docentes que hasta hace poco solo podían dar clases a un reducido y cierto número de estudiantes, confinados en un aula de cuatro paredes, podrán dar clases ahora a un infinito números de estudiantes, no solo en el ámbito de la jurisdicción de la Universidad, sino en cualquier parte del mundo, los docentes tendrán la necesidad de ir a las ya obsoletas instalaciones universitarias, para arregla una "menudencia".

Al comienzo de esta investigación, Covid-19: Retos y Oportunidades en el Proceso de Acreditación de las Universidades y luego de consultas tanto bibliográficas, Hemerográficas, electrónicas, documentales etc. Fueron surgiendo una serie de datos e informes de varios autores, con respecto a este tema; ahora bien; la crisis causada por el Covid-19, ha traído como consecuencia una revalorización de la vida académica y de la educación como un todo, la virtualización de las instituciones de educación también ha hecho de la acreditación un tema que cobra mayor relevancia hoy en día, porque es a través de ésta, en donde la calidad y la excelencia educativa presuponen uno de los baluartes más apreciados por las Universidades y por ende de la sociedad en general.

Es desde allí; en donde han surgido una serie de cuestionamiento tales como ¿qué parámetros se deben establecer para medir la excelencia académica, en tiempos de educación virtual producto de la crisis generada por el Covid-19? ¿Cuál será el método utilizado para la acreditación institucional Universitaria, en una educación a distancia?, a los largo de este escrito se ha tratado de conocer los caminos, las aristas y la significancia de la acreditación en el ámbito Universitario, las respuestas a todas estas preguntas tendrán relevancia, en tanto y en cuanto, estas agencias, estos organismos, asuman de manera clara, diáfana, y con toda responsabilidad los nuevos cambios que son inexorables, de la valoración que hagan ellos, de su compromiso con la excelencia y la calidad de la enseñanza en las instituciones de educación superior, se podrá hacer un balance en el futuro de su rol como custodios de la acreditación. 
Por otra parte; son mucho los desafíos que ha traído la pandemia del Covid-19, pero a la vez muchos retos y oportunidades, por ejemplo, la acreditación deberá lidiar con la masificación de la educación de manera virtual, ya no son círculos cerrados de discusión en un aula, serán miles de estudiantes conectados, desde cualquier parte del mundo, escuchando y viendo una clase virtual. Con respecto; a los docentes, éstos convertirán cualquier espacio reducido en un aula virtual, prácticamente es el traslado de la universidad a la cochera de su casa, ósea su casa convertida en una pequeña universidad, en donde los requerimientos serán mínimos, ahora bien; todo este cambio en los encargados de impartir clases a los estudiantes, trae aparejado una serie cuestiones a resolver por parte de los organismos encargados de dar la respectiva acreditación a éste tipo de estudio, que debido a la pandemia se han hecho cotidiano y es que; como medir el rendimiento académico de una institución de educación virtual, en donde todo su recurso humano y sus estudiantes se encuentra disgregado, ya no; en el país de origen de la universidad, sino en cualquier parte del mundo, como saber que parámetros aplicar para medir la calidad educativa y la excelencia académica.

Hay que mencionar además; que la bioseguridad como mecanismo de control para evitar el contagio originado por el Covid-19, hace que el distanciamiento social sea la norma y no la excepción, obligando a las universidades a mantener el personal mínimo dentro de las instalaciones, de allí; las dificultades de los ente de acreditación, en cómo; valorar la calidad educativa, con una planta profesoral y estudiantil no presente en las aulas, y a distancia considerables del ente educativo, este surgimiento del higienismo como política de máxima seguridad y que ha dado origen a la diversificación de la mayoría de carreras virtuales y por ende de la educación a distancia, es lo que impone a los organismos encargados de dar las acreditaciones a las instituciones Universitarias, fijar nuevos caminos, nuevos métodos, nuevas reglas, para lograr que la educación superior que ha tomado un nuevo rumbo, con la virtualización en ciernes, logre la eficiencia, la eficacia, la calidad y la excelencia educativa.

Se debe agregar; que son más y más los desarrollos tecnológicos que cada día impactan sobremanera a la sociedad como un todo, y donde las instituciones de educación superior adquieren mayor relevancia, entre éstas: la inteligencia artificial, la robótica, y a punto de irrumpir, uno de los últimos adelantos tecnológicos, las redes 5G, lo que se ha dado a llamarse "el internet de las cosas", son innovaciones en donde la academia, con sus organismos de acreditación, tendrán la última palabra para lograr la calidad y la excelencia educativa, porque; son innumerables las nuevas carreras que han ido surgiendo, acorde con las nuevas realidades, y a la par de todos estos adelantos tecnológicos la universidad no puede ni debe quedar relegada, lo mismo los organismos encargadas de su acreditación.

Es necesario recalcar; que todo este cambio tecnológico, toda esta reclusión originada por el Covid-19, viene a representar el surgimiento de lo que han comenzado a llamar algunos estudiosos, sobre 
ésta realidad, "el ser virtual”, que no es más que aquel individuo que ya no necesita salir de su casa, porque toda su vida, toda su actividad, será realizada telemáticamente, de hecho un altísimo porcentaje de las cosas que se hacen hoy en día, son realizadas a través de la red. Además todo este entramado virtual se ha acelerado de manera espectacular con la llegada de Covid-19, dentro de todo esto;el rol que le toca jugar a las instituciones de educación Superior y los órganos de acreditación, vienen a ser de nuevo el semillero de esta novísima forma educativa que está naciendo, ésta digitalización del proceso académico, deberán dar respuestas más oportunas, eficientes y eficaces al conglomerado al cual sirve y a la sociedad en general, porque toda éste conocimiento, toda ésta inmediatez, estará a solo un clic de la ciudadanía.

Finalmente; todo lo que se ha generado producto del Covid-19, todas las trasformaciones que están en proceso, todas aquellas decisiones que han tomado las autoridades de las instituciones de educación superior, para la formación y educación de los estudiantes como futuros profesionales, están dirigidos a formar ciudadanía y para que sean ellos capaces de resolver aquellos problemas públicos, derivados de la vida en sociedad y como no; la humanidad misma, con herramientas capaces de dar respuestas a las próximas pandemias o al cambio climático, como por ejemplo, que cada día serán más recurrentes y todo esto; con el concurso de las academias de educación superior y los organismos que acrediten estos nuevos conocimientos con la excelencia y la calidad educativa. 


\section{REFERENCIAS BIBLIOGRAFICAS}

Alonso, Randy. (2019). Informe Global Digital 2019: Más de un millón de nuevos internautas diarios en el mundo el pasado año. [Ver Online]. http://www.citmatel.cu/noticias/informe-global-digital-2019mas-de-un-millon-de-nuevos-internautas-diarios-en-el-mundo-el

Díaz, Eduardo; Jezibel, Jiménez; Joer, Velazco. (2000). Las TIC y la educación superior del siglo XXI Discutir los temas sobre las TIC y la educación superior del siglo XXI- [Ver Online]. https://lasticedusupsigloxxi.wordpress.com/paradigmas-educativos/

López, Francisco. (2003). La transformación de los procesos de acreditación: retos y recomendaciones. SegreraUniversidade de Salamanca. [Ver Online] file://D:/USUARIO/Desktop /Curso\%20Basico\%20Online \%20Microsoft/1.\% 20Microsoft\%20Excel/ Elementos\%20Recibidos/Dialnet-LaTransformaci

Pierre, Levi. (1999) ¿Qué es lo virtual? Barcelona. [Ver Online]. Paidós- http://espacio.uned.es/fez/eserv/bibliuned:Ried-2001-numero1-2090/Documento.pdf

Rincón, Gustavo. (2020). Retos de calidad en tiempos de COVID, 19--- 2 abril, 2020.(Jefe de la Oficina de Gestión y Aseguramiento de la Calidad, profesor catedrático; Consejero Comisión Nacional Intersectorial de Aseguramiento de la Calidad de la Educación Superior CONACES). [Ver Online].https://noticias.uniquindio.edu.co/retos-de-calidad-en-tiempos-de-covid-19/

Viegas, Juan; Fronti, Luisa, Suárez, Suárez, Elsa; Scvone, Graciela; Fronti, Inés (2001). Educación virtual como complemento de la educación formal y continuada. Trabajo "Interamericano" presentado en la XXIV ConferenciaInteramericana de Contabilidad. Punta del Este, Uruguay. [Ver Online]. http://bibliotecadigital.econ.uba.ar/download/cya/cya_v7_n14_02.pdf

Zapata, Miguel. (2015). Pensamiento computacional: Una nueva alfabetización digital ComputationalThinking: A New Digital Literacy. Universidad de Murcia. España [Ver Online]. https://www.um.es/ead/red/46/zapata.pdf 